Nephrologe 2017 $\cdot 12: 80-81$

DOI 10.1007/s11560-017-0140-3

๑) Springer Medizin Verlag Berlin 2017

CrossMark

\author{
J. Hoyer ${ }^{1} \cdot$ H. Haller ${ }^{2}$ \\ 'Klinik für Innere Medizin und Nephrologie, Universitätsklinikum der Philipps-Universität Marburg, \\ Marburg, Deutschland \\ ${ }^{2}$ Klinik für Nieren- und Hochdruckerkrankungen, Medizinische Hochschule Hannover, Hannover, \\ Deutschland
}

\title{
Update Hypertonie
}

In der Hypertensiologie ist in den letzten zwei Jahren wieder viel geschehen. Im vorliegenden Heft haben wir versucht wichtige Entwicklungen und Neuerungen für Sie zusammenzufassen.

Ein wichtiges Thema sind die Zielblutdruckwerte bei Patienten mit Hypertonie. Diese Diskussion ist durch einige Publikationen neu entfacht worden. In den vergangenen Jahren gab es mehrere qualitativ hochwertige Studien zur notwendigen Intensität der Blutdrucksenkung. Im Rahmen dieser Studien fiel wiederholt auf, dass trotz positiver kardiovaskulärer Effekte eine intensive Blutdrucksenkung mit einer signifikanten Verschlechterung der Nierenfunktion einherging (siehe Artikel von J. Hoyer). Hier muss die Nephrologie eventuell umdenken; das oft propagierte „so niedrig wie möglich“ für Patienten mit Nierenerkrankungen muss kritisch hinterfragt werden. Es scheint dringend notwendig zu sein, dass die Nephrologie durch kontrollierte Studien die Ursachen der renalen Funktionsverschlechterung bei intensiver Blutdrucksenkung untersucht, mögliche Risikogruppen identifiziert und die Bedeutung der Nierenfunktionsverschlechterung in Relation zur Risikoreduktion für kardiovaskuläre Erkrankungen stellt.

Die Einführung der SGLT2 (,sodium-glucose cotransporter 2“)-Inhibitoren wird nicht nur in der Diabetologie Therapieverbesserungen ermöglichen, sondern insbesondere für die Nephrologie wichtige Erkenntnisse erbringen. Es ist nicht übertrieben, die Einführung dieser Substanzklasse als einen der wichtigen Meilensteine in der Therapie von Patienten mit Nierenkrankheiten zu betrachten. In seinem Beitrag stellt J. Menne hierzu die neuesten Daten zusammen.
Neben der besseren Blutzuckereinstellung konnte in den ersten Studien mit SGLT2-Inhibitoren gezeigt werden, dass im Langzeitverlauf auch eine Verbesserung der Nierenfunktion und eine anhaltende moderate Blutdrucksenkung erreicht werden. Die Ursachen für die Blutdrucksenkung sind noch ungeklärt. Sie ist einerseits auf die diuretische Wirkung mit konsekutiver Reduzierung des Volumenhochdrucks zurückzuführen. Sie könnte jedoch auch durch die Reduzierung vasopressorischer renaler Systeme wie dem Renin-AngiotensinAldosteron-System (RAAS) mitbewirkt werden.

》) Die Hypertonie gewinnt in
der langfristigen Therapiefüh-
rung von Systemerkrankungen
und Tumorerkrankungen
zunehmend an Bedeutung

Zwei Gebiete, auf denen die Hypertonie zunehmend an Bedeutung in der langfristigen Therapieführung erhält, sind die Systemerkrankungen und die Tumorerkrankungen. In der Tumortherapiekonnte in den letzten zehn Jahren durch neuartige Therapeutika die Überlebenszeit von Tumorpatienten entscheidend verbessert werden. Wie im Artikel von I. Grgic et al. dargestellt wird, haben diese neuen Therapieformen jedoch Nebeneffekte auf die Vasoregulation und können zu deutlichen Blutdruckerhöhungen führen. Deshalb ist es für klinisch tätige Hypertensiologen wichtig, auf diesem Gebiet frühzeitig Kenntnisse zu erlangen, um die behandelnden Onkologen in der langfristigen Therapie ihrer Patienten effektiv unterstützen zu können. System- 
erkrankungen werden häufig durch die Entwicklung einer arteriellen Hypertonie kompliziert. Dies ist insbesondere der Fall, wenn, wie bei Vaskulitis oder beim hämolytisch-urämischen Syndrom (HUS), eine Endothelschädigung im Vordergrund steht. Wie J. Becker et al. in ihrem Artikel aufzeigen, können Hypertensiologen bei solchen Systemerkrankungen wichtige Hinweise für die spezifische Abstimmung von Diagnostik und antihypertensiver Therapie geben sowie krisenhafte Blutdruckentgleisungen vermeiden helfen.

Die Präeklampsie ist nach wie vor die häufigste Komplikation im Verlauf einer Schwangerschaft. Die zellulären und molekularen Mechanismen der Erkrankung sind in den letzten Jahren besser verstanden worden. Dies erlaubt nicht nur ein besseres pathophysiologisches Verständnis der Erkrankung, sondern hat auch neue diagnostische Möglichkeiten eröffnet. Außerdem zeichnen sich neue Therapiestrategien (Plasmapherese, Immunabsorption) für diese bedrohliche Erkrankung ab. M. Golic und R. Dechend stellen in ihrem Beitrag diese neuen Forschungen und ihre Auswirkungen auf Diagnostik und Therapie der Präeklampsie vor.

Wir hoffen mit diesem Schwerpunktheft Ihre hypertensiologische Expertise weiter zu vertiefen und Ihnen Impulse für Ihre tägliche Praxis geben zu können und wünschen Ihnen viel Freude beim Lesen.

Ihr

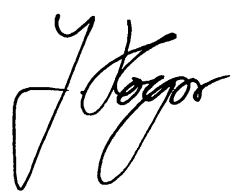

J. Hoyer

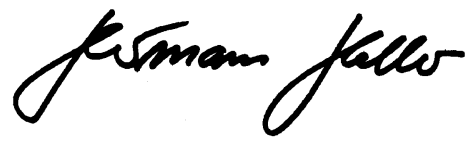

H. Haller

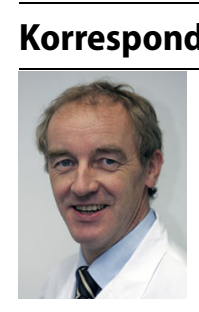

\section{Künstliche Beta-Zellen aus menschlichen Nierenzellen entwickelt}

Prof. Dr. J. Hoyer

Klinik für Innere Medizin und Nephrologie, Universitätsklinikum der Philipps-Universität Marburg Baldingerstraße 1, 35043 Marburg, Deutschland hoyer@med.uni-marburg.de

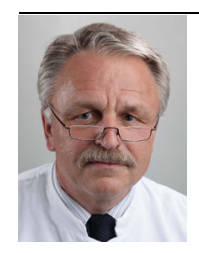

Prof. Dr. H. Haller

Klinik für Nieren- und Hochdruckerkrankungen, Medizinische Hochschule Hannover Carl-Neuberg-Str. 1, 30625 Hannover, Deutschland Haller.Hermann@ mh-hannover.de

Interessenkonflikt. J. Hoyer und H. Haller geben an, dass kein Interessenkonflikt besteht.
Mit einem verhältnismäßig einfachen Ansatz haben Schweizer Forscher künstliche Beta-Zellen hergestellt, die alles können, was natürliche Beta-Zellen auf der Bauchspeicheldrüse leisten: Sie messen die Glukosekonzentration im Blut und sie bilden Insulin, um den Blutzuckerspiegel zu senken. Hierzu verwendeten die Forschenden eine Zelllinie, die auf menschlichen Nierenzellen beruht, sog. HEK-Zellen. Die Forscher nutzten die natürlichen Glukose-Transportproteine und Kalium-Kanäle in der Membran der HEK-Zellen. Diese erweiterten sie um einen spannungsabhängigen Kalziumkanal sowie um Gene zur Produktion von Insulin und GLP1.

In den künstlichen Beta-Zellen befördert das natürliche Glukose-Transportprotein der HEK-Zellen Glukose aus dem Blut ins Zellinnere. Sobald der Blutzuckerspiegel eine gewisse Schwelle überschreitet, schließen sich die Kalium-Kanäle. Dadurch kippt die Spannungsverteilung an der Membran, die Kalzium-Kanäle öffnen sich und das einströmende Kalzium löst eine in die HEK-Zellen eingebaute Signalkaskade aus an deren Ende die Produktion und Ausschüttung von Insulin respektive GLP1 stehen.

Die Wissenschaftler testeten die künstlichen Beta-Zellen vorerst in Mäusen. Dabei entpuppten sich die Zellen als äußerst leistungsfähig. Sie funktionierten besser und länger als alle bisherigen Lösungen. In diabetischen Mäusen implantiert, produzierten die modifizierten HEK-Zellen während drei Wochen zuverlässig und in ausreichenden Mengen die Blutzuckerspiegel regulierenden Botenstoffe.

Ob die künstlichen Beta-Zellen auch im Menschen verwendet werden können, müssen nun weitere klinische Tests zeigen.

\section{Originalpublikation:}

Xie M et al. (2016) Beta-cell-mimetic designer cells provide closed-loop glycemic control. Science.

DOI: $10.1126 /$ science.aaf4006

Quelle: Eidgenössische Technische Hochschule Zürich (www.ethz.ch) 